SFA and SAD

\title{
How do I look?
}

Self-focused Attention during a Video Chat of Women with Social Anxiety (Disorder)

Noortje Vriends* ${ }^{*,}, 1,2$,Yasemin Meral ${ }^{* 1,3}$, Javier A. Bargas-Avila ${ }^{1,4}$, Christina Stadler $^{2}, \&$ Susan M. Bögels ${ }^{5}$ 


\begin{abstract}
We investigated the role of self-focused attention (SFA) in social anxiety (disorder) in an ecologically valid way. In Experiment 1 high $(n=26)$ versus low $(n=25)$ socially anxious single women between 18 - 30 years had a video ("Skype") conversation with an attractive male confederate, while seeing themselves and the confederate on-screen. The conversation was divided in four phases: (I) warm-up, (II) positive (confederate was friendly to the participant), (III) critical (confederate was critical to the participant), and (IV) active (participant was instructed to ask questions to the confederate). Participant's SFA was measured by eye-tracked gaze duration at their own image relative to the confederates' video image and other places at the computer screen. Results show that high socially anxious participants were more self-focused in the critical phase, but less self-focused in the active phase than low socially anxious participants. In Experiment 2 women diagnosed with SAD ( $n$ $=32)$ and controls $(n=30)$ between $18-30$ years conducted the same experiment. Compared to controls participants with SAD showed increased SFA across all four phases of the conversation, and SFA predicted increased self-rated anxiety during the conversation.

In conclusion, in subclinical social anxiety SFA is high only when the interaction partner is critical, whereas instructions to ask questions to the confederate reduces subclinical socially anxious' SFA, while clinical SAD is characterized by heightened self-focused attention throughout the interaction. Results support theories that social anxiety disorder is maintained by SFA, and imply that interventions that lower SFA may help prevent and treat social anxiety disorder, but that self-focused attention can also be adaptive in certain types of interaction, such as when receiving compliments.
\end{abstract}


SFA and SAD

Keywords: social anxiety disorder; self-focused attention; arousal; cognitive model; eyetracking 


\section{Introduction}

Clark and Wells' (1995) cognitive model of social anxiety disorder (SAD) predicts that self-focused attention (SFA) plays a central role in maintaining social anxiety. Since the publication of this model SFA and social anxiety has been repeatedly investigated. Studies that experimentally manipulated SFA (e.g. with mirrors, video cameras, an audience) report mixed results. Some studies found SFA to be related to social anxiety (e.g. Meral, Vriends, \& Meyer, 2013; Woody \& Rodriguez, 2000; Zou, Hudson, \& Rapee, 2007), whereas others failed to show an association between social anxiety and SFA (e.g., Bögels, Rijsemus, \& De Jong, 2002, see for review: Bögels \& Mansell, 2004). Probe-detection paradigms investigating attention towards internal cues (e.g. physical cues, such as heart rate) versus external cues (e.g. a visual probe of household objects or emotional faces) found an attentional bias toward internal cues (indicating SFA) in speech-anxious individuals (Deiters, Stevens, Hermann, \& Gerlach, 2013; Mansell, Clark, \& Ehlers, 2003) and in socially anxious individuals (Mills, Grant, Judah, \& White, 2014; Pineles \& Mineka, 2005). More specifically, this internal attentional bias was only found in women (Mansell et al., 2003) and in socially anxious participants with an independent (versus interdependent) self-construal (Vriends et al., 2016). Self-report studies on SFA show more congruent results, namely higher SFA in high socially anxious individuals compared to low socially anxious individuals (e.g. Alden \& Mellings, 2004; Bögels \& Lamers, 2002; Perowne \& Mansell, 2002; Spurr \& Stopa, 2003; Woody, 1996; Woody \& Rodriguez, 2000).

Cognitive behavioral treatments for SAD that included exercises to reduce SFA show to be effective in reducing SFA and social anxiety, as measured by self-report questionnaires (e.g. McManus et al., 2009; Schreiber, Heimlich, Schweitzer, \& Stangier, 2015). Task concentration training, an intervention that solely focuses on reducing SFA, was found to be more effective than applied relaxation (Bögels, 2006) or exposure in vivo (Mulkens, Bögels, de Jong, \& Louwers, 2001) in reducing fear of showing bodily symptoms in patients with 
social anxiety (disorder), and reduced self-reported SFA was shown to mediate these superior results (Bögels, 2006).

In sum, there is ample evidence that social anxiety is related to SFA from questionnaire and intervention research, but experimental research provided mixed results. . A reason for the mixed picture in experimental research might be the challenge to measure SFA validly within social experiments. Another explanation is that subjective SFA report might be biased, as it is the same reporter reporting SFA and social anxiety. Also, participants might have difficulties being aware of the focus of their attention during social interaction. Therefore it is worthwhile to use implicit assessment methods for SFA. Probe-detection tasks do this, but have other disadvantages such as interfering with the social task itself. Another challenge is that social situations are dynamic, continuously demanding different tasks. Thus, there is need to explore more ecologically valid and creative ways to implicitly measure SFA within social situations.

In the present study, we measured SFA using eye-tracking methods within a social situation - in a "Skype" conversation (the Swiss Social Interaction Task (SSIT)) with a confederate in which the participant underwent four phases: (I) 'warm-up', (II) 'positive' (confederate was friendly to the participant), (III) 'critical' (confederate put the participant in the center of attention and was critical to the participant), and (IV) 'active' (participant had to ask questions to the confederate). This way, the social interaction involved positive and negative social challenges as well as being evaluated (the confederate is asking questions) and evaluating (the participant is asking questions). Eye-tracking methods have proven to be an adequate method to asses attentional processes in social anxiety (e.g. Buckner, Maner, \& Schmidt, 2010; Gamble \& Rapee, 2010; Schofield, Johnson, Inhoff, \& Coles, 2012; Wieser, Pauli, Alpers, \& Muhlberger, 2009), but none of these studies investigated SFA. In Experiment 1 we pilot the paradigm with high versus low socially anxious women. In 
SFA and SAD

Experiment 2 we tested the paradigm in a clinically relevant sample of women with social anxiety disorders versus controls.

\section{Experiment 1}

Socially anxious people are assumed to suffer from heightened SFA. They direct too much attention to themselves during social interactions and have little attention for other people, their task or their environment (e.g. Bögels, 2006; Clark \& Wells, 1995; Rapee \& Heimberg, 1997). Therefore we assume that watching one's own video image on a computer screen during an online-chat situation would indicate heightened SFA. Chatting online is nowadays a common way of online-dating or peer-to-peer communication and therefore an ecologically valid situation (Smith \& Anderson, 2016). Eye-tracking allows a non-invasive direct and continuous measurement of visual attention within the situation without interfering in the current social task. We invited high- end low socially anxious single women for a social experiment. They were instructed to have a real-time video conversation with a male participant, who actually was a confederate. SFA during the conversation was measured directly by eye-tracking (focusing on self-image versus on image of confederate or on other places at the computer screen) and self-rated by participants after the conversation. Before and after the conversation, participants also rated their level of current stress. We expected (I) increased SFA (measured by self-report and eye-tracking) during the conversation in the high socially anxious group, and (II) a significant correlation between eye-tracked SFA and current stress after the conversation. Explorative we analyzed the effect of the phases of the conversation on SFA and other-focused attention next to self-focused attention.

\section{Material and Methods}

\subsection{Participants}

Single women with an age between 18 and 30 years participated in a video conversation study. They were recruited with advertisements on the University of Basel study advertisement webpage and with posters at local University restaurants. We recruited only 
SFA and SAD

female individuals due to found sex effects with respect to SFA in previous studies (Mansell et al., 2003; Vriends et al., 2016). We selected women with high versus low social anxiety through an online screening - the Social Interaction Anxiety Scale (Mattick \& Clarke, 1998; german version: Stangier, Heidenreich, Berardi, Golbs, \& Hoyer, 1999), which was added at local advertisement webpages. Individuals with a SIAS-score above 40 and below 20 were invited for the experiment. One woman was excluded from the analyses due to outlier results (more than 2 SD's from the mean on relevant variables). The study-sample ( $n=51)$ was median-split on the scores of the Social Phobia Scale (which was filled out at the beginning of the experiment) for high and low social anxiousness groups. The two groups (high socially anxious $n=25$ and low socially anxious $n=26$ ) did not differ in age and education (see Table 2).

\subsection{Materials}

Social anxiety. The German version of the Social Phobia Scale (SPS: Mattick \& Clarke, 1998; German version: Stangier et al., 1999) was used to measure social anxiety. The SPS assesses anxiety in performance situations and includes 20 items rated on a 5-point scale ranging from 0 (not at all) to 4 (very much). A total score (ranging from 0 to 80 ) consists of the sum of all items. Scores above 24 on the SPS indicate social anxiety disorder (Heimberg, Mueller, Holt, Hope, \& Liebowitz, 1992). The questionnaire showed high internal consistency for the present study (Cronbach's alpha $=.91$ ).

Instruction for the Swiss Social Interaction Task (SSIT). The experimenter informed the participant that she would have a video conversation with a male participant (who was our confederate, but the participant was led to believe that the male counterpart was also a participant), who was sitting in another experimental room. The task was to get to know each other. The experimenter told the participant that the confederate was instructed to ask her some questions in the first 4 minutes to get to know her better. After that she would have 


\section{SFA and SAD}

also 4 minutes to get to know him by asking questions. Participants were also informed that their gaze would be tracked during the video conversation.

Confederates. Seven attractive male confederates, of similar ages than the female participants, were involved in this study. They were briefly informed about the study (they were told that our study was about attentional processes during a conversation with different phases of social stress) and were blind to the participants' condition (high versus low socially anxious) and to the study's hypotheses. Confederates received 1,5-day training before the start of the study on how to induce the different phases during conversation. Confederates were paid according to Swiss norm for student assistant honorarium.

Social interaction with the confederate - the SSIT. The participant sat at a comfortable chair about $80 \mathrm{~cm}$ behind a computer screen and was led to believe that they were having a video conversation with another study participant. During the conversation, the participant could see the video image of the confederate and the same-sized image of herself displayed on their computer screen (see Figure 1). The side of the own video-image (right or left) was counterbalanced. The video conversation was composed of four phases: (I) warm-up phase, in which neutral questions were asked (II) positive phase, during which the confederate was playfully flirty (III) critical phase, during which the participant was felt to be negatively evaluated and (IV) active phase, when the participant led the conversation (see a detailed description of the phases in Table 1). For each phase the confederate followed a script with a battery of questions and verbal/nonverbal behaviors.

The content of the first three phases were not communicated to the participants - they only were aware of two phases that were used as the cover story of the paradigm: the phase in which the confederate was asking questions (warm-up, positive, and critical phase) and the phase in which the participant was asking questions (active phase). The experimenter instructed the confederate regarding timing through a small display at his computer screen. 
Self-focused attention (SFA). SFA was measured directly by tracking participants gaze behavior (hereafter referred to as eye-tracked SFA to distinguish it from self-rated SFA). During the conversation with the confederate, an eye-tracker (SMI) was placed below the monitor and recorded eye movements with $60 \mathrm{~Hz}$ on a 1,680×1,050-pixel screen. Before starting the conversation an eye-tracker calibration was performed. Eye-tracking data were analyzed using the software BeGaze 3.3 (SMI). Mean fixation times for the four phases for each participant were calculated. Fixations were defined as eye movements within $1^{\circ}$ of visual field for a minimum duration of $80 \mathrm{~ms}$. SFA was calculated for each phase by the percentage of time that the participant observed her own video image relative to the duration of watching the confederate or watching at other places at the screen during the standardized phases (warm-up $1 \mathrm{~min}$; positive 1,5 min; critical 1,5 min; active $4 \mathrm{~min}$ ).

Self-rated SFA was measured with the Self-Focused Attention Scale (SFA: Bögels, Alberts, \& de Jong, 1996) translated to German and rephrased from trait SFA to retrospectively ask about subjective SFA during conversation (state SFA). The state SFA consists of 11 items on a 5-point scale from 0 (never) to 4 (very often). Total scores range from 0 to 44, with higher scores indicating higher SFA. The internal consistency of the State SFA in this study was high, with Cronbach's alpha $=.85$.

Other-focused attention (OFA). Other-focused attention was defined as the percentage of time that the participant watched the confederate's video image relative to the duration of watching themselves or watching other places of the screen during the phases of the conversation. Positive values mean higher SFA or OFA.

Current stress. To investigate participants' current stress before and immediately after the video conversation, they filled out four $100 \mathrm{~mm}$ visual analogue scales (VASs) to measure current nervousness, anxiety, self-confidence (reversed), and arousal, running from "not at all" to "extremely", the mean score indicating current stress. 
SFA and SAD

Credibility check. At the end of the experiment, the experimenter asked the participants if they noted something special in the experiment and/or about the male participant (our confederate). Three independent raters coded the answers into "probably believed that confederate was a participant" or "probably did not believe that the confederate was participant". Interrater reliability was $97 \%$. Differences in scores were discussed with the first author and then rated

Integrity check. To assess integrity of the confederates, two independent observers rated the confederates' behavior towards the participants during the video conversation. Using 5-point Likert-scales they answered how friendly the confederate interacted, how easily the confederate chatted without stumbling (speech flow), and if he behaved in line with the instructions for the phases of the conversation. If rates of the raters differed $>1$ point on the Likert-scale, a third rater $(\mathrm{YM})$ rated the video $(\mathrm{n}=2)$.

\subsection{Procedure}

The experiment took place at the social behavior and physiology lab at the Faculty of Psychology of the University of Basel and was approved and accepted by the ethical commission of Basel (EKBB, 338/08). After signing informed consent (all participants who came to the experiment signed) participants completed questionnaires about sociodemographic details and the Social Phobia Scale (SPS). Then the experimenter informed them about the video conversation with the male participant (our confederate). After this instruction participants filled out the current stress visual analogue scales (VASs) for their current nervousness, anxiety, self-confidence, and arousal, performed the eye-tracker calibration, and started the conversation. After the conversation, participants filled out again the VAS question about how currently stressed they felt. They also completed the State Self-focused Attention Scale (State SFA) to retrospectively ask about subjective SFA during the conversation and the current stress VASs. Finally, participants were asked about the credibility of the experiment (see above), were debriefed, and dismissed. 
SFA and SAD

\subsection{Statistical analyses}

Data preparation and data checking were conducted using SPSS 23. Group differences were analyzed using $t$-tests or Chi-quadrat-tests. Group differences for eye-tracked SFA and OFA were analyzed with GLM for repeated measures. The within subject factor was SFA or OFA during the four phases of the conversation (warm-up, positive, critical, and active) and the between-subject factor was group (high versus low socially anxious). The eye-tracked SFA and OFA data were log transformed to reach normality. Correlations between eyetracked SFA, self-rated SFA, OFA and current stress were analyzed with bivariate correlations. An alpha level of $<.05$ was used for all analysis.

\section{Results}

\subsection{Credibility and integrity of confederate}

Independent raters rated 45 participants (88\%) as believing that the conversation partner was a participant. The high and low socially anxious participants did not differ in this regard $\left(\chi^{2}=.67, p=.413\right)$. External raters rated the integrity of the confederates in the high versus low socially anxious group similar $(F(1,49)=1.55, p=.219, \eta 2=.03)$ indicating that the confederates treated both groups equally.

\subsection{Self-focused attention during social interaction}

In contrast to the hypothesis, no main effect for group occurred $(p>.05)$, indicating that socially anxious women did not focus more to themselves across all phases of the conversation. A significant main effect for phase $(F(3,147)=4.70, p=.004, \eta 2=.09)$ showed that women looked most at themselves during the critical phase and least at themselves during the active phase. A significant interaction between group and phase occurred $(F(3,147)=3.23, p=.028, \eta 2=.06)$, see Figure 2, showing that women with high social anxiety focused more on themselves in the critical phase and less on themselves in the active phase, compared to women with low social anxiety. As hypothesized, high socially anxious women rated their SFA during the conversation higher than low socially anxious 
SFA and SAD

women (high socially anxious, $M=1,50, S D=.59$; low socially anxious, $M=.99, S D=.59$; $t(49)=-2.70, p=.010, d=.77)$. Self-rated SFA correlated in the low social anxiety group negatively with eye-tracked SFA during the critical $(r(24)=-.39, p=.049)$ and the active phase $(r(24)=-.40, p=.041)$. In the high social anxiety group correlations were positive but not significant $(p ’ s>.05)^{1}$.

\subsection{Other-focused attention during the social interaction}

No main effect for group occurred $(p=.582)$, indicating that overall high socially anxious women did not look differently at their interaction partner. A main effect for phase $(F(3,147)=13.73, p=.000, \eta 2=.22)$ showed that women looked at the confederate the most during the warm-up and active phase, see Figure 3. No interaction between group and phase occurred $(p=.109)$, see Figure 3. In both groups SFA and OFA significantly negatively correlated during all phases (high social anxiety: $p$ 's $\leq .016$; low social anxiety: $p$ ' $\leq .001)^{1}$.

\subsection{Association between eye-tracked self-focused attention and current stress.}

In the high social anxiety group eye-tracked SFA during the warm-up, critical and active phase correlated with higher current anxiety after the conversation (resp. $r(23)=.56, p$ $=.003 ; .478, p=.016 ; .45, p=.023)$, whereas the other correlations between eye-tracked SFA during the conversation and current stress (nervousness, arousal, self-confidence) before and after the conversation were not significant $(p \text { 's }>.05)^{1}$. In the low social anxiety group no significant correlations occurred $(p ’ s>.05)^{1}$.

\section{Discussion}

The results of Experiment 1 show that the paradigm worked well in imitating a social situation for young adult women, in which self-focused attention was measured by eyetracking (gaze duration at own video display). While high socially anxious women, as hypothesized, self-reported heightened self-focused attention during the conversation compared to low socially anxious women, we did not find the expected overall higher eye-

\footnotetext{
${ }^{1}$ Correlation coefficients can be given upon request.
} 


\section{SFA and SAD}

tracked focus on the self in high socially anxious women compared to low socially anxious women. Several explanations can be given for this difference in outcome between selfreported state SFA and objectively measured SFA. Looking at their own image (knowing this is the image that the conversation partner sees) might have another, positive, effect on low socially anxious single women when meeting an attractive man than on their high socially anxious counterparts. That is, as low socially anxious individuals have more positive expectancies about how others view them, they may get a more positive feeling when looking at themselves through the eyes of the attractive man, than high socially anxious individuals will. This explanation is consistent with the lack of correlation between eye-tracked SFA and current stress in low socially anxious women. Related, our high socially anxious participants may have seen observable stress symptoms (e.g., red spots in neck or face) while looking at themselves, which may have made them avoiding looking at their own image further, compared to high socially anxious women. An alternative explanation for the difference in outcome between self-reported state SFA and objectively measured SFA, is that self-rated SFA was not specified for each conversation phase as objectively measures SFA was, and thus participants were not able to report the differences that they experienced which we will discuss below.

We found that high socially anxious women looked more at themselves when the man became critical, whereas, interestingly, low socially anxious women looked less at themselves when he became critical relatively to the other phases of the conversation. We might speculate that low socially anxious women focus less on their own image during the critical phase to protect themselves. Interestingly, when they had the lead over the social situation (when they were instructed to actively ask questions), high socially anxious women focused less on themselves compared to low socially anxious women relatively to the other phases of the conversation. Being instructed to actively ask questions to the other person may have helped high socially anxious women to focus more on the task (getting to know the other 
SFA and SAD

person) rather than themselves and as such decrease their self-focus, which is consistent with interventions in which individuals with social anxiety are trained to focus more on the task and the environment rather than on themselves during social situations (Bögels, 2006). However, as group by phase differences in other-focused attention were not significant, we cannot conclude that the task-focus indeed made high socially anxious women focus more on the other person, but that it did help them to focus less on themselves.

To conclude, with a "Skype" dating-like conversation paradigm we were able to measure self- and other-focused attention on the spot and the paradigm showed that the focus of attention depends on the demands of the conversation and whether the participant is high or low socially anxious.

\section{Experiment 2}

\section{Introduction}

Experiment 1 showed that the new chat conversation paradigm is ecologically valid, works well, and that eye-tracking is a useful method to measure SFA. The next relevant step was to replicate the experiment with a clinical sample suffering from social anxiety disorder. In line with Experiment 1 and with the cognitive model, we hypothesized (I) increased overall self-rated and eye-tracked SFA during the conversation in participants with SAD compared to control participants. Based on the findings of experiment 1 we hypothesized (II) increased eye-tracked SFA in participants with SAD particularly during the critical phase compared to controls. Finally, we hypothesized that (III) eye-tracked SFA predicts self-reported current stress after the conversation. Explorative, we analyzed eye-tracked other-focused attention.

\section{Material and Methods}

\subsection{Participants}

The sample was recruited with online advertisement at the University of Basel website and at several websites providing information about SAD. Also flyers with information about the study were distributed on regional postings and in general medical institutions. Again, we 


\section{SFA and SAD}

recruited only female individuals due to sex effects in previous studies (Mansell et al., 2003; Vriends et al., 2016). All interested participants were first screened in a telephone interview, which was developed for the present experiment. The screening comprised questions about 1) social situations in which participants felt anxious, 2) impairment because of social anxiety, 3) fears about what could happen in that social situation, 4) physical symptoms, and 5) medication. Only potential participants with SAD or controls were invited to the diagnostic interview. Inclusion criteria for the SAD group were age between 18 and 30 years, fluent in German, primary social anxiety disorder, and heterosexual. Exclusion criteria for the SAD group were a lifetime history of DSM-IV (Diagnostic and Statistical Manual of Mental Disorders, 4th ed.; American Psychiatric Association, 1994) criteria for schizophrenia, bipolar disorder, dementia, current major depressive disorder, current substance and alcohol abuse, and current use of any recreational drugs or current medication on psychoactive drugs (including selective serotonin reuptake inhibitors). The exclusion criteria for the control group were the same as for the SAD group plus a lifetime history of anxiety and mood disorders, homosexual. They were matched with the SAD group on age, ethnicity, and education. All interested individuals were first screened in a telephone interview. Potential participants were then invited to a diagnostic interview. SAD and comorbid disorders were diagnosed with a clinical interview (DIPS, Diagnostisches Interview bei psychischen Störungen) for DSM-IV Axis I disorders (Schneider \& Margraf, 2011). The interrater reliability of the diagnostic interview was high with kappa .95. Interviewers for the DIPS were intensively trained and supervised by the author (NV). On the basis of diagnoses, we composed the SAD and control groups. The SAD sample consisted of 32 participants with a primary diagnosis of SAD and the control group consisted of 30 participants. Three women were excluded from the analyses due to outlier results (more than 2 SD's from mean on relevant variables). The sample details are presented in Table 3. No group differences were found for age and education (see Table $3)$. 
SFA and SAD

\subsection{Materials}

For materials see section 2.2 of Experiment 1, except for the following improvements and supplements:

Social anxiety. Beside the Social Phobia Scale, the German version of the Social Interaction Anxiety Scale (SPS/SIAS: Mattick \& Clarke, 1998; German version: Stangier et al., 1999) was also used to measure social anxiety, which assesses anxiety in social interactions. The SIAS includes 20 items rated on a 5-point scale ranging from 0 (not at all) to 4 (very much). A total score (ranging from 0 to 80 ) consists of the sum of all items. Both questionnaires had high internal consistency in the present study with a Cronbach's alpha of .95 for the SPS and .88 for the SIAS.

Confederates. Five attractive male confederates of similar age as the participants were involved in the SSIT. Training and information about the study was identical to Experiment 1.

Credibility check. We performed the credibility and the integrity check as in Experiment 1, and included two new items, namely 1) the question if the participant thought whether the other participant (confederate) had prepared his questions for the conversation, and 2) on a 10-point scale participants rated the authenticity of the conversation and the confederate.

Eye-tracked self- and other-focused attention. We improved these data by marking the exact beginnings and ends of the phases of the conversation, as we observed that the lengths of the phases of the conversation varied in Experiment 1 marginally. Therewith, we used the exact durations of the phases of the conversation (instead of standardized duration for phases) for SFA and OFA.

\subsection{Procedure}


SFA and SAD

Ethical permission (EKBB 338/08) was obtained and participants signed an informed consent ${ }^{2}$. Besides the small differences in participant selection the procedure was identical to Experiment 1 .

\subsection{Statistical analyses}

Statistical methods and analyses were identical to Experiment 1.

\section{Results}

\subsection{Credibility and integrity of the confederates}

The mean rating of the authenticity of the conversation was $6.90(S D=2.06)$ on a 10 point scale. $96.8 \%$ of the participants reported that they noticed nothing special about the confederate and the conversation. $85.5 \%$ did not believe not that the confederate had prepared his questions for the conversation. No significant differences between the groups were found on the credibility and authenticity items ( $p$ 's $>.05)$. The confederates' behavior toward SAD and control participants did not differ, $F(3,56)=2.01, p=.123, \eta^{2}=.10$.

\subsection{Eye-tracked SFA and self-rated SFA during the conversation}

In line with the hypothesis, we found a main effect of group for eye-tracked SFA, $F(1$, $60)=4.17, p=.046, \eta^{2}=.06$ (see Figure 4), indicating that participants with SAD observed their own image more than the image of the confederate across all phases of the conversation compared to control participants. A main effect of phase was found, $F(3,180)=7.87, p=$ $.000, \eta^{2}=.12$ (see Figure 4), indicating that SFA was higher in the warm-up and the critical phase compared to the positive and the active phase. No interaction between group and phase occurred, $F(3,180)=.59, p=.621, \eta^{2}=.01$.

As expected, the SAD group rated their state SFA during the conversation significantly higher than the control group, effect size being very large (SAD: $M=25.97, S D$ $=6.86$; control: $M=11.20, S D=7.05), t(60)=8.36, p<.001, d=2.13$. In the controls self-

\footnotetext{
${ }^{2}$ Heart rate and skin conductance was also measured, but not presented because it is behind the scope of this paper.
} 
SFA and SAD

rated state SFA correlated negatively $(r(28)=-.42, p=.019)$ and in the SAD group positively $(r(30)=.364, p=.040)$ with eye-tracked SFA during the critical phase.

\subsection{Eye-tracked OFA during the conversation}

We found a main effect for phase, $F(1,180)=12.30, p=.000, \eta 2=.17$ (see Figure 5), showing that participants looked most at the confederate during the warm-up and the active phase. Neither the main effect for group $(p=.699)$ nor the interaction between group and phase $(p=.436)$ was significant. SFA and OFA did not correlate during all four phase in both groups $(p ’ s>.05)^{1}$.

\subsection{Association between eye-tracked self-focused attention and self-reported current stress}

Eye-tracked SFA during the critical phase correlated marginally with lower selfreported current self-confidence $r(30)=.34, p=.058$ ) after the conversation in the SAD group. Other correlations between self-report current stress items and eye-tracked SFA during the phases were not significant ( $p$ 's $>.1)$.

\section{Discussion}

Results of Experiment 2 show that, in line with expectations, young adult women with SAD were more self-focused (eye-tracked and self-rated) during a "Skype" conversation with an attractive male confederate than healthy controls, which propose that individuals with SAD are more self-focused than control individuals in social situations. In participants with SAD eye-tracked SFA during the conversation was associated with decreased self-reported current self-confidence after the conversation, indicating that SFA lowers self-confidence.

The results of Experiment 2 support attentional models of SAD (Clark \& Wells, 1995; Rapee \& Heimberg, 1997).

In line with Experiment 1, we see that SFA is not stable, though varies significantly across the phases of the conversation. SFA is highest at the beginning of a conversation with a novel person (an attractive person of the opposite sex), and while being criticized or put in the 
center of attention in a stressful way, and lowest when being complimented and when instructed to take an active role in the conversation.

In contrast to Experiment 1, we found that all participants (SAD and control) show increased SFA when being criticized. Also the finding that participants with SAD are generally more self-focused than control participants as measured with eye-tracking is in contrast to Experiment 1. There are several explanations for the different results between the two experiments. One explanation is that the eye-tracking data of Experiment 2 are more reliable, because we could take the exact lengths of the phases of the conversation, whereas in Experiment 1 we could only take the standardized length of the phases of the conversation. Another explanation is that the confederates of Experiment 1 and 2 (that were not the same) were activating different reactions. A third explanation, as the women in Experiment 1 were single, while those in Experiment 2 not necessarily, is that being complimented (flirting) had a different effect on participants in Experiment 1. Still another explanation is that the clinical nature of the Experiment 2 participants (social anxiety disorder diagnosis) accounts for the different results. Possible too is that the low socially-anxious group of Experiment 1 was an extreme group with not as-usual behavior, whereas the Experiment 2 control group was a normal control group (absence of social anxiety disorder).

\section{General Discussion}

We used a new paradigm to examine the focus of attention during a social interaction with different phases of social stress (warm-up, positive, critical, and active) for high versus low socially anxious single young women (Experiment 1) and in young adult women with a current social anxiety disorder (SAD) versus without any lifetime anxiety or depressive disorder (Experiment 2). In Experiment 1 socially anxious participants showed increased eyetracked SFA when they were criticized by their conversation partner, but decreased eyetracked SFA when they were instructed to actively ask questions to the conversation partner, compared to low socially anxious participants. In Experiment 2 we replicated the study with a 
clinical SAD and control sample, and found that eye-tracked SFA was increased in women with SAD compared to healthy controls across all phases of the conversation. Further, we found in women with SAD that eye-tracked SFA during the conversation was associated with feeling currently nervous before conversation and less self-confident after the conversation. Another interesting finding of the present experiments is that in both eye-tracked SFA was found to vary strongly across the phases.

To our knowledge the present study is the first that succeeded in measuring SFA using eye-tracking in an ecologically valid social interaction without interfering with the social task (such as reacting as fast as possible at stimuli). Therewith, our result of increased eye-tracked SFA in patients with SAD goes in line with and exceeds experimental research measuring SFA with dot-probe-detection paradigms that found faster reaction times on internal (selffocused) probes than external (other- or task-focused) probes in anticipation to social stress situation (Deiters et al., 2013; Mansell et al., 2003; Mills et al., 2014; Pineles \& Mineka, 2005). The additional benefit of measuring SFA within the social situation without interfering in the social task is that we can investigate the direct effect of SFA within different social stress levels (warm-up, positive, critical, and active phase) and different social tasks (e.g. answering and asking questions) in the social situation. In line with cognitive and attention models of social anxiety (disorder) our results show that eye-tracked SFA is increased in SAD and associated with currently feeling nervous. The finding that for both socially anxious (disordered) and control participants eye-tracked SFA seems to vary depending on the social task at hand is a new finding. During an active phase, where the individual is challenged to take initiative and thereby also has more control over the social situation (in our case by asking questions to the confederate in order to get to know him better), eye-tracked SFA appears to be relatively lowest and OFA relatively highest. A possible explanation for this finding is that during this phase the participants' primary task was to evaluate the confederate 


\section{SFA and SAD}

and therefore they looked more at the confederate, whereas during the other phases participants might have felt more evaluated, and therefore looked more at themselves.

If our results can be replicated, the instruction to patients with SAD of focusing more at the task and less at themselves could be enriched with the message to variably focus on the task and on the self in correspondence with the task requirements. Also in other psychological disorders, current literature underlines the variable use of strategies. The importance of a variable use of strategies has been proven by some studies. For example, the findings of Joormann and Gotlib (2010) suggest that individual differences in the use of emotionregulation strategies play an important role in depression. Also many psychophysiological studies show that flexibility (for example in cardiovascular responsibility; heart rate variability) is a predictor for mental health (Thayer \& Lane, 2000) and that cognitive processes and physiological variability are related (e.g. Thayer, Hansen, Saus-Rose, \& Johnsen, 2009; Williams et al., 2015).

Opposed to present models of SAD we found different patterns of results between the women with clinical diagnosis of social anxiety disorder and the high socially anxious subclinical women, that is, only increased eye-tracked SFA in the critical phase for high socially anxious women and decreased SFA in the active phase, versus increased SFA across phases of the conversation in women with social anxiety disorder. Although methodological explanations may account for this difference in results of both experiments, as discussed in section 8 , a true difference between high social anxiety and social anxiety disorder in selffocused attention may exist. One could speculate that when social anxiety is still within manageable levels, self-focused attention is only a problem when being criticized or put in the center of attention, and becoming socially active is a helpful way to redirect attention away from the self and manage social anxiety levels. When heightened self-focused attention is generalized across different types of social interactions (our phases), and simply becoming active is not any longer enough to counteract self-focused attention, social anxiety might 


\section{SFA and SAD}

become a pervasive pattern (disorder). This might represent a pathway from high social anxiety through SFA to social anxiety disorder. Future studies might investigate this pattern and its implications for treatment.

The following limitations should be noted in the current study. First, our findings are only applicable to females. Other studies investigating SFA found gender effects (e.g. Mansell et al., 2003; Vriends et al., 2016), thus, replication of these findings with males need to be investigated. Second, the conversation through video might have influenced SFA. The presence of a video image might reduce typical self-focus, increase it, or interact with diagnostic status. Bögels et al. (2002) and Hofmann and Heinrichs (2003) used mirrors in their studies to induce SFA, which next to heightening self-focus may have acted as an external source of information that socially anxious individuals used to correct their exaggerated negative self-images (e.g., finding out while watching in the mirror that their feeling of blushing is hardly visible). So the complex issue with enhancing self-focused attention by (video or mirror) self-image is that it next to heightening self-focus provides objective information about once appearance which is important in the modulation of the mental representation of the self. Third, the effect of phases could also be an effect of time (such as novelty, habituation, concentration) because the four phases of the social interaction were not randomized. However, probably, in case of a time effect SFA would linearly decline during the conversation. Fourth, we used a laboratory getting-acquainted interaction, and the extent to which this design generalizes to real-life-situations needs to be established.

The present study has clinical potential. If the present results are replicated, eyetracking is a useful instrument for measuring SFA in social situations and might become a useful intervention method such as by providing feedback in interventions targeting attention problems in social anxiety. For example, it would be scientifically relevant to investigate eyetracked SFA during a performance task (seeing oneself, the audience and the task at hand, e.g. the PowerPoint slide) and to show the results of the eye-tracking to the person with the SAD. 
SFA and SAD

Also it would be innovative to feedback the eye-tracking to the person during the task - in that way attention could directly be trained. Our results show that SFA strongly varied during different phases of the conversation. Thus, it might be helpful to the therapist to know that higher SFA might be adaptive in certain interactions (such as when being complimented), and that reducing SFA to zero might be maladaptive. Emotional and attentional responses that are consistent with environmental demands represent adaptive emotional regulation and promote physical and mental health (Thayer \& Lane, 2000).

In conclusion, in subclinical social anxiety self-focused attention is high only when the interaction partner is critical, whereas instructions to ask questions to the confederate reduces subclinical socially anxious' self-focused attention, while clinical SAD is characterized by heightened self-focused attention throughout the interaction. Results support theories that social anxiety disorder is maintained by self-focused attention, and imply that interventions that lower self-focused attention may help prevent and treat social anxiety disorder, but that self-focused attention can also be adaptive in certain types of interaction, such as when receiving compliments. 


\section{References}

Alden, L. E., \& Mellings, T. M. (2004). Generalized Social Phobia and social judgments: the salience of self- and partner-information. Journal of Anxiety Disorders, 18(2), 143157.

Bögels, S. M. (2006). Task concentration training versus applied relaxation, in combination with cognitive therapy, for social phobia patients with fear of blushing, trembling, and sweating. Behaviour Research and Therapy, 44(8), 1199-1210. doi:

10.1016/j.brat.2005.08.010

Bögels, S. M., Alberts, M., \& de Jong, P. J. (1996). Self-consciousness, self-focused attention, blushing propensity and fear of blushing. Personality and Individual Differences, 21(4), 573-581.

Bögels, S. M., \& Lamers, C. T. (2002). The causal role of self-awareness in blushing-anxious, socially-anxious and social phobics individuals. Behaviour Research and Therapy, 40(12), 1367-1384.

Bögels, S. M., Rijsemus, W., \& De Jong, P. J. (2002). Self-focused attention and social anxiety: The effects of experimentally heightened self-awareness on fear, blushing, cognitions, and social skills. Cognitive Therapy and Research, 26(4), 461-472.

Buckner, J. D., Maner, J. K., \& Schmidt, N. B. (2010). Difficulty Disengaging Attention from Social Threat in Social Anxiety. Cognitive Therapy and Research, 34(1), 99-105. doi: 10.1007/s10608-008-9205-y

Clark, D. M., \& Wells, A. (1995). A cognitive model of social phobia. In R. G. Heimberg, M. R. Liebowiz, D. A. Hope, \& F. R. Schneier (Eds.), Social phobia: Diagnosis, assessment, and treatment (pp. 69 - 93). New York: The Guilford Press. 
SFA and SAD

Deiters, D. D., Stevens, S., Hermann, C., \& Gerlach, A. L. (2013). Internal and external attention in speech anxiety. Journal of Behavior Therapy and Experimental Psychiatry, 44(2), 143-149.

Gamble, A. L., \& Rapee, R. M. (2010). The time-course of attention to emotional faces in social phobia. Journal of Behavior Therapy and Experimental Psychiatry, 41(1), 3944. doi: 10.1016/j.jbtep.2009.08.008

Heimberg, R. G., Mueller, G. P., Holt, C. S., Hope, D. A., \& Liebowitz, M. R. (1992). Assessment of Anxiety in Social-Interaction and Being Observed by Others - the Social-Interaction Anxiety Scale and the Social Phobia Scale. Behavior Therapy, 23(1), 53-73.

Hofmann, S. G., \& Heinrichs, N. (2003). Differential Effect of Mirror Manipulation on SelfPerception in Social Phobia Subtypes. Cognitive Therapy and Research, 27(2), 131142.

Joormann, J., \& Gotlib, I. H. (2010). Emotion regulation in depression: Relation to cognitive inhibition. Cognition \& Emotion, 24(2), 281-298. doi: Pii 917893204

$10.1080 / 02699930903407948$

Mansell, W., Clark, D. M., \& Ehlers, A. (2003). Internal versus external attention in social anxiety: an investigation using a novel paradigm. Behaviour Research and Therapy, 41(5), 555-572.

Mattick, R. P., \& Clarke, J. C. (1998). Development and validation of measures of social phobia scrutiny fear and social interaction anxiety. Behaviour Research and Therapy, $36(4), 455-470$.

McManus, F., Clark, D. M., Grey, N., Wild, J., Hirsch, C., Fennell, M., . . Manley, J. (2009). A demonstration of the efficacy of two of the components of cognitive therapy for social phobia. Journal of Anxiety Disorders, 23(4), 496-503. doi:

10.1016/j.janxdis.2008.10.010 
SFA and SAD

Meral, Y., Vriends, N., \& Meyer, A. H. (2013). Self-image, self-focused attention, and social performance in a social interaction situation: What is relevant for social anxiety? presented at EABCT congress 2013.

Mills, A. C., Grant, D. M., Judah, M. R., \& White, E. J. (2014). The influence of anticipatory processing on attentional biases in social anxiety. Behavior Therapy, 45(5), 720-729.

Mulkens, S., Bögels, S. M., de Jong, P. J., \& Louwers, J. (2001). Fear of blushing: Effects of task concentration training versus exposure in vivo on fear and physiology. Journal of Anxiety Disorders, 15(5), 413-432. doi: Doi 10.1016/S0887-6185(01)00073-1

Perowne, S., \& Mansell, W. (2002). Social anxiety, self-focused attention, and the discrimination of negative, neutral and positive audience members by their non-verbal behaviours. Behavioural and Cognitive Psychotherapy, 30(1), 11-23. doi: $10.1017 / \mathrm{S} 1352465802001030$

Pineles, S. L., \& Mineka, S. (2005). Attentional biases to internal and external sources of potential threat in social anxiety. Journal of Abnormal Psychology, 114(2), 314-318.

Rapee, R. M., \& Heimberg, R. G. (1997). A cognitive-behavioral model of anxiety in social phobia. Behaviour Research and Therapy, 35(8), 741-756.

Schneider, S., \& Margraf, J. (2011). DIPS diagnostisches Interview bei psychischen Störungen. Berlin: Springer.

Schofield, C. A., Johnson, A. L., Inhoff, A. W., \& Coles, M. E. (2012). Social anxiety and difficulty disengaging threat: Evidence from eye-tracking. Cognition \& Emotion, $26(2), 300-311$.

Schreiber, F., Heimlich, C., Schweitzer, C., \& Stangier, U. (2015). Cognitive therapy for social anxiety disorder: the impact of the "self-focused attention and safety behaviours experiment" on the course of treatment. Behavioural and Cognitive Psychotherapy, 43(2), 158-166. doi: 10.1017/S1352465813000672 
SFA and SAD

Smith, A., \& Anderson, M. (2016). 5 facts about online dating. from http://www.pewresearch.org/fact-tank/2016/02/29/5-facts-about-onlinedating/

Spurr, J. M., \& Stopa, L. (2003). The observer perspective: effects on social anxiety and performance. Behaviour Research and Therapy, 41(9), 1009-1028.

Stangier, R., Heidenreich, T., Berardi, A., Golbs, U., \& Hoyer, J. (1999). Assessment of social phobia by the Social Interaction Anxiety Scale and the Social Phobia Scale. Zeitschrift Fur Klinische Psychologie-Forschung Und Praxis, 28(1), 28-36.

Thayer, J. F., Hansen, A. L., Saus-Rose, E., \& Johnsen, B. H. (2009). Heart rate variability, prefrontal neural function, and cognitive performance: the neurovisceral integration perspective on self-regulation, adaptation, and health. Annals of Behavioral Medicine, 37(2), 141-153. doi: 10.1007/s12160-009-9101-z

Thayer, J. F., \& Lane, R. D. (2000). A model of neurovisceral integration in emotion regulation and dysregulation. Journal of Affective Disorders, 61(3), 201-216.

Vriends, N., Bolt, O. C., Meral, Y., Meyer, A. H., Bögels, S. M., \& Wilhelm, F. H. (2016). Does self-focused attention in social anxiety depend on self-construal? Evidence from a probe detection paradigm. Journal of Experimental Psychopathology, 7(1), 18-30.

Wieser, M. J., Pauli, P., Alpers, G. W., \& Muhlberger, A. (2009). Is eye to eye contact really threatening and avoided in social anxiety?--An eye-tracking and psychophysiology study. Journal of Anxiety Disorders, 23(1), 93-103.

Williams, D. P., Cash, C., Rankin, C., Bernardi, A., Koenig, J., \& Thayer, J. F. (2015). Resting heart rate variability predicts self-reported difficulties in emotion regulation: a focus on different facets of emotion regulation. Front Psychol, 6, 261. doi: 10.3389/fpsyg.2015.00261

Woody, S. R. (1996). Effects of focus of attention on anxiety levels and social performance of individuals with social phobia. Journal of Abnormal Psychology, 105(1), 61-69. 
SFA and SAD

Woody, S. R., \& Rodriguez, B. F. (2000). Self-focused attention and social anxiety in social phobics and normal controls. Cognitive Therapy and Research, 24(4), 473-488.

Zou, J. B., Hudson, J. L., \& Rapee, R. M. (2007). The effect of attentional focus on social anxiety. Behaviour Research and Therapy, 45(10), 2326-2333. 
Table 1.

Content of the four phases of the video conversation - the Swiss Social Interaction Task

\begin{tabular}{|c|c|c|c|}
\hline $\begin{array}{l}\text { Number of } \\
\text { Phase }\end{array}$ & Title of Phase & $\begin{array}{l}\text { Length in } \\
\text { minutes }\end{array}$ & Description \\
\hline 1 & Warm-up & 1 & $\begin{array}{l}\text { The confederate first asked the } \\
\text { participant's name, age and some neutral } \\
\text { questions about study/work-situation, } \\
\text { living situation, or the actual weather. }\end{array}$ \\
\hline 2 & Positive & 1,5 & $\begin{array}{l}\text { The confederate was friendly and showed } \\
\text { that he liked the participant by making } \\
\text { compliments such as "that sounds nice- } \\
\text { could you tell me more about that?" and } \\
\text { "you are cool". His behavioral facial and } \\
\text { verbal expressions were kind and playful } \\
\text { flirty (not intimidating) to the participant. }\end{array}$ \\
\hline 3 & Critical & 1,5 & $\begin{array}{l}\text { The confederate was critical. He tried to } \\
\text { make the participant feel socially anxious, } \\
\text { or a little negatively evaluated. He } \\
\text { focused on negative characteristics, } \\
\text { making comments such as "Tell me about } \\
\text { your negative characteristics" or "That } \\
\text { was embarrassing!" or asking the } \\
\text { participant to sing: "What is your favorite } \\
\text { song? I don't know that song; can you } \\
\text { sing it for me?" }\end{array}$ \\
\hline 4 & Active & 4 & $\begin{array}{l}\text { The participant was invited to lead the } \\
\text { conversation and could direct questions to } \\
\text { their conversation partner (the } \\
\text { confederate). The confederate was } \\
\text { instructed to answer the questions during } \\
\text { this phase }\end{array}$ \\
\hline
\end{tabular}


Table 2

Characteristics of the Participants in the High Socially Anxious and Low Socially Anxious Group in Experiment 1

\begin{tabular}{|c|c|c|c|c|c|}
\hline \multirow[b]{2}{*}{ Variable } & \multicolumn{2}{|c|}{ Group } & \multirow[b]{2}{*}{$\chi^{2}$} & \multirow[b]{2}{*}{$t$} & \multirow[b]{2}{*}{$p$} \\
\hline & $\begin{array}{l}\text { High socially anxious } \\
\qquad(N=25)\end{array}$ & $\begin{array}{l}\text { Low socially anxious } \\
\qquad(N=26)\end{array}$ & & & \\
\hline Mean age, in years $(S D)$ & $23.27(3.317)$ & $23.24(4.18)$ & & .03 & .978 \\
\hline $\begin{array}{l}\text { Education ( } n \text {, compulsory education/high } \\
\text { school/university) }\end{array}$ & $2 / 18 / 6$ & $4 / 17 / 4$ & 1.076 & & .584 \\
\hline \multicolumn{6}{|l|}{ Social anxiety ${ }^{\mathrm{a}}$} \\
\hline SPS, mean $(S D)$ & $32,88(9.79)$ & $10.92(5.97)$ & & 9.71 & .001 \\
\hline
\end{tabular}

Note. SPS = Social Phobia Scale;

${ }^{\mathrm{a}} \mathrm{Scores}$ above 24 on the SPS indicate social phobia. 
Table 3

Characteristics of the Participants in the Social Anxiety Disorder and Control Groups in Experiment 2

\begin{tabular}{|c|c|c|c|c|c|}
\hline \multirow[b]{2}{*}{ Variable } & \multicolumn{2}{|c|}{ Group } & \multirow[b]{2}{*}{$\chi^{2}$} & \multirow[b]{2}{*}{$t$} & \multirow[b]{2}{*}{$p$} \\
\hline & $\begin{array}{l}\text { Social anxiety disorder } \\
\qquad(N=32)\end{array}$ & $\begin{array}{l}\text { Control } \\
(N=30)\end{array}$ & & & \\
\hline Mean age, in years $(S D)$ & $22.41(3.90)$ & $23.00(2.99)$ & & 0.67 & .506 \\
\hline $\begin{array}{l}\text { Education ( } n, \text { compulsory education/high } \\
\text { school/university) }\end{array}$ & $6 / 19 / 7$ & $5 / 14 / 11$ & 1.675 & & .433 \\
\hline \multicolumn{6}{|l|}{ Social anxiety ${ }^{\mathrm{a}}$} \\
\hline SPS, mean $(S D)$ & $41.66(13.43)$ & $11.30(9.92)$ & & 10.07 & .001 \\
\hline SIAS, mean $(S D)$ & $37.06(11.12)$ & $18.40(6.85)$ & & 7.90 & .001 \\
\hline \multicolumn{6}{|c|}{ No. $(\%)$ with comorbid anxiety disorders in the social anxiety disorder group } \\
\hline Panic disorder & $1(3.1 \%)$ & - & & & \\
\hline Agoraphobia & $2(6.3 \%)$ & - & & & \\
\hline Specific phobia & $8(25 \%)$ & - & & & \\
\hline Generalized anxiety disorder & $7(21.9 \%)$ & - & & & \\
\hline Posttraumatic stress disorder & $1(3.1 \%)$ & - & & & \\
\hline Somatic symptom disorder & $1(3.1 \%)$ & - & & & \\
\hline
\end{tabular}

Note. SPS = Social Phobia Scale; SIAS = Social Interaction Anxiety Scale.

${ }^{\mathrm{a}} \mathrm{Scores}$ above 34 on the SIAS and above 24 on the SPS indicate social phobia. 


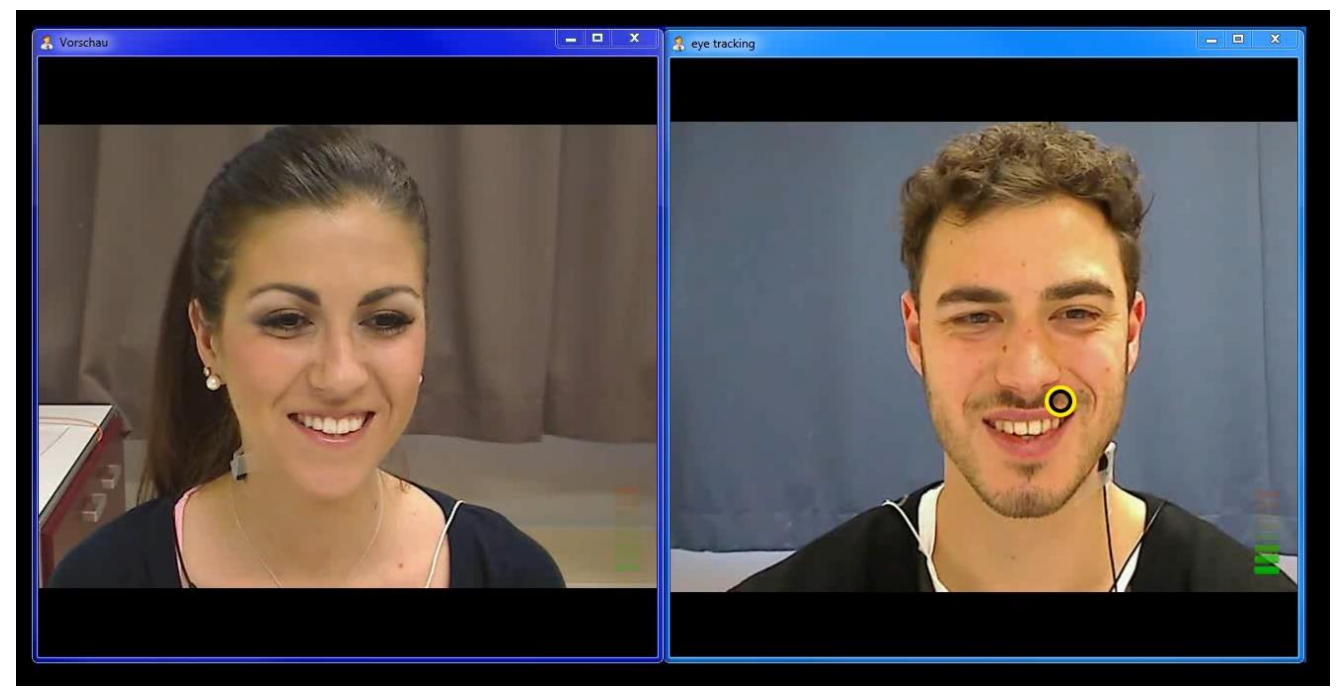

Figure 1. Video conversation with the confederate. Participants observed their own video image and a same-sized video image of the confederate on the computer screen. 


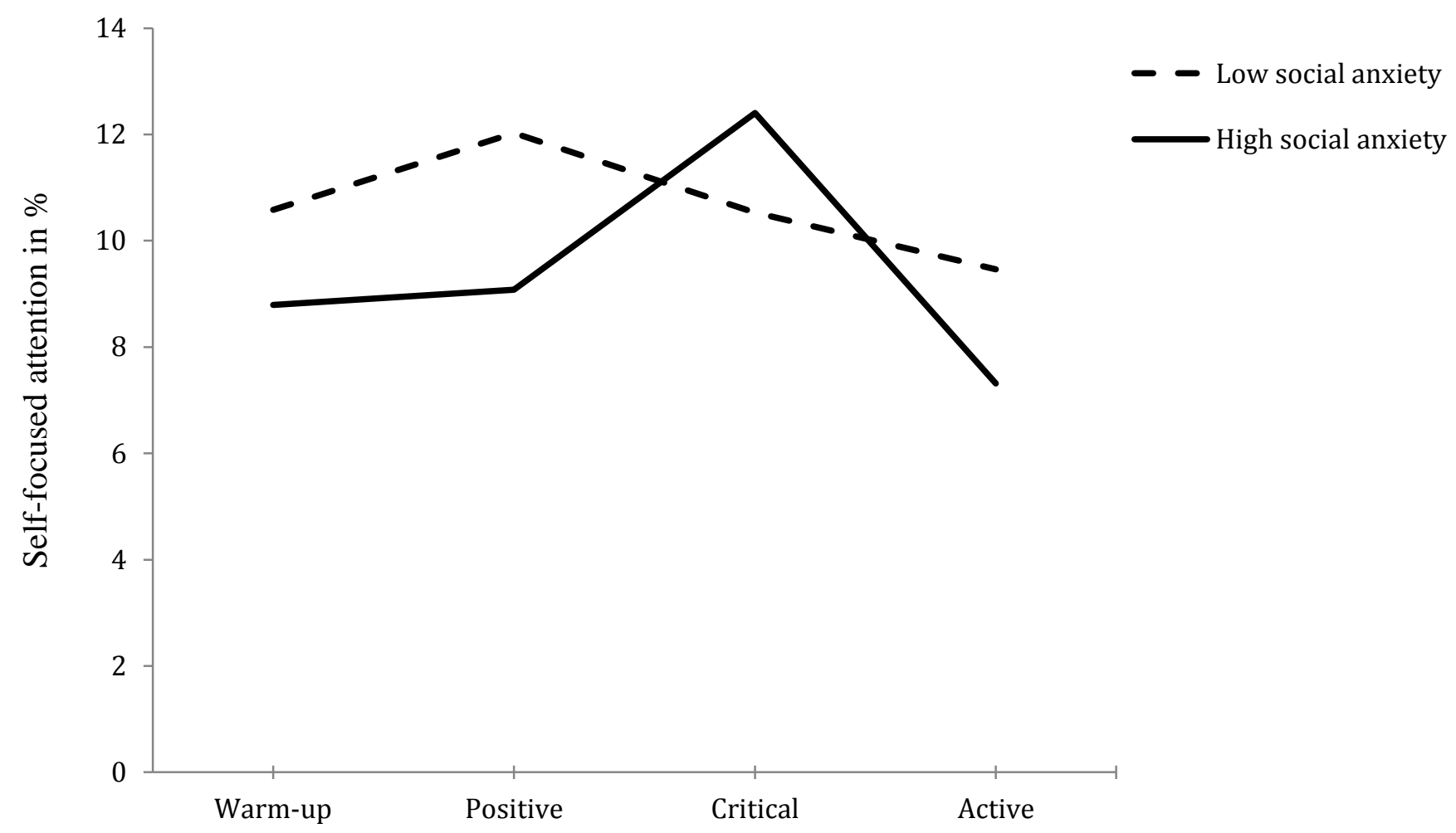

Figure 2. Eye-tracked SFA during the four phases of the conversation with the confederate in Experiment 1. 


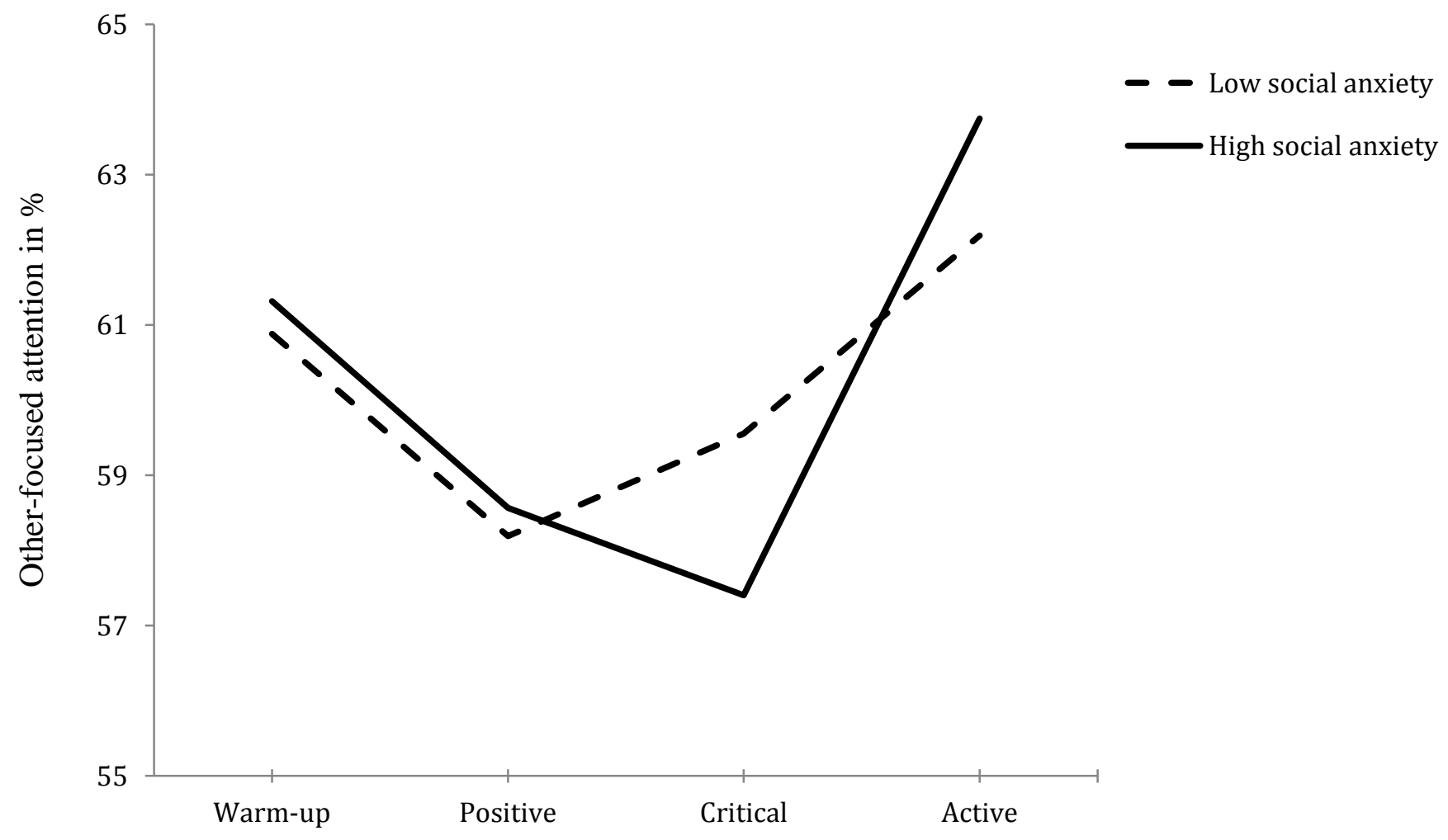

Figure 3. Eye-tracked OFA during the four phases of the conversation with the confederate in Experiment 1. 


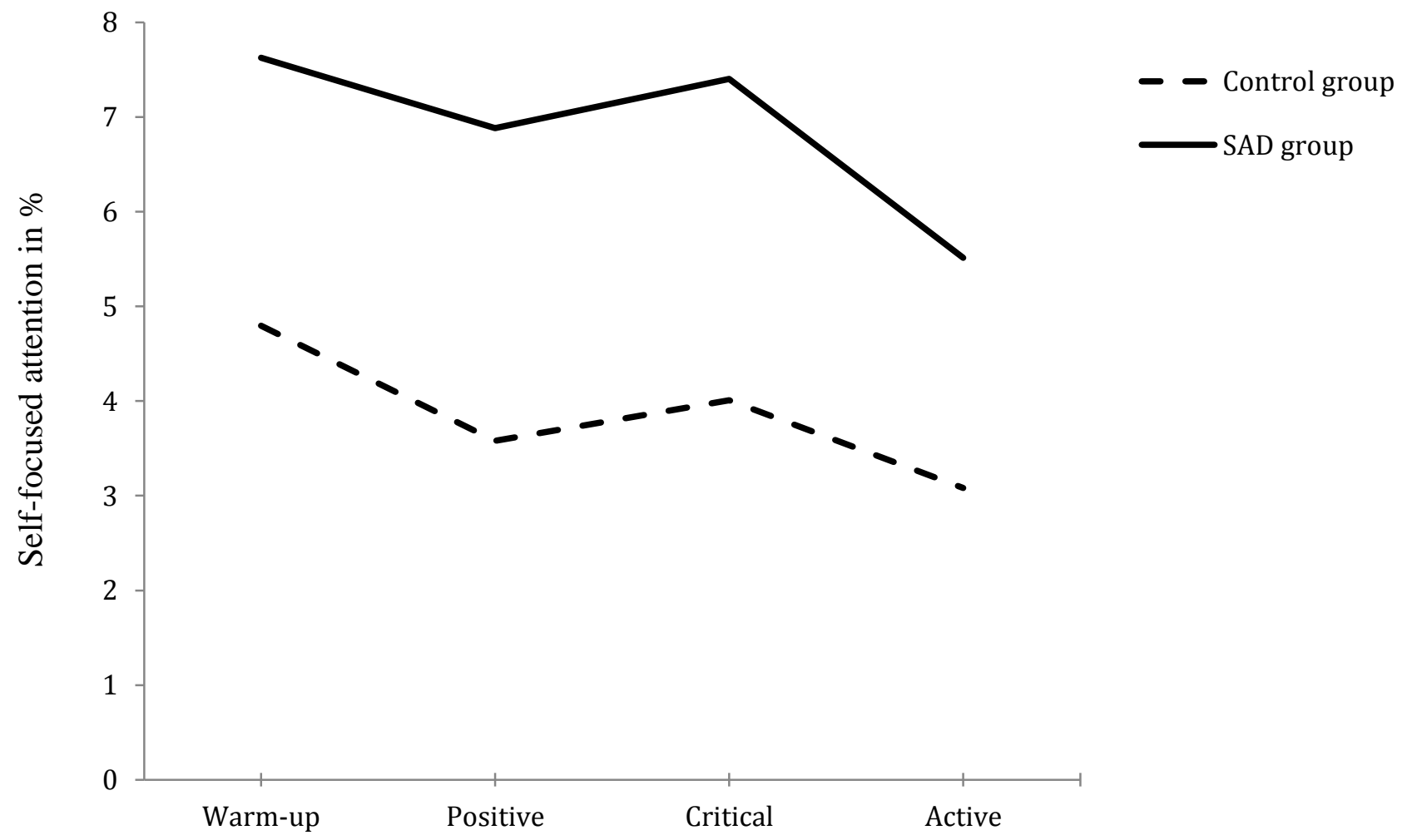

Figure 4. Eye-tracked SFA during the four phases of the conversation with the confederate in Experiment 2. 


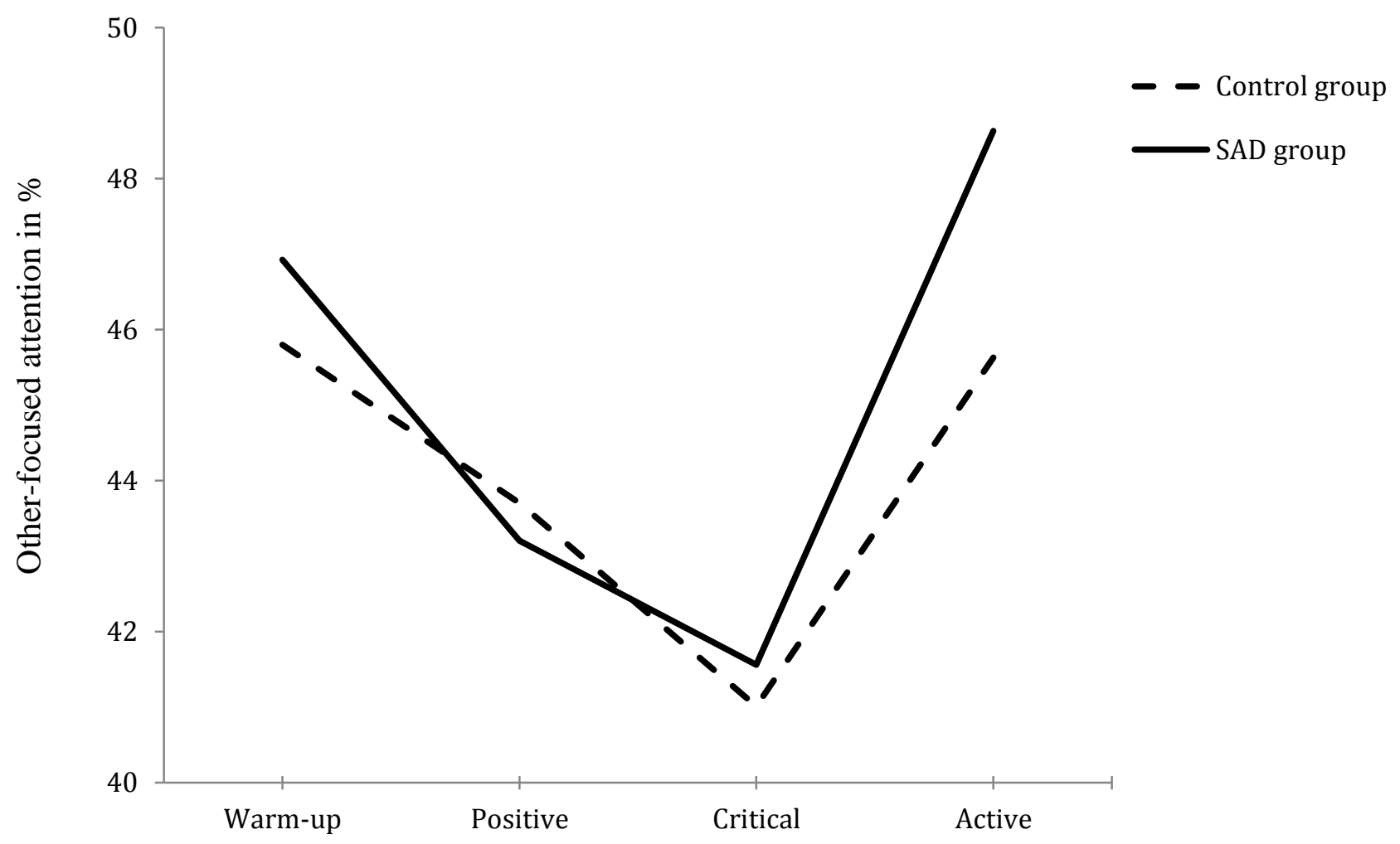

Figure 5. Eye-tracked OFA during the four phases of the conversation with the confederate in Experiment 2. 